\title{
SOME PROPERTIES OF WEAK SOLUTIONS OF NONLINEAR SCALAR FIELD EQUATIONS
}

\section{Li Gongbao}

\section{Introduction and the main results}

In this paper, we study some properties of the weak solutions of the following nonlinear scalar field equations

$$
\begin{gathered}
-\sum_{i=1}^{N} \frac{\partial}{\partial x_{i}}\left(|\nabla u|^{p-2} \frac{\partial u}{\partial x_{i}}\right)+c(x)|u|^{q-2} u=f(x, u), \\
u \in E=\left\{u \in L^{q}\left(R^{N}\right) \mid \frac{\partial u}{\partial x_{i}} \in L^{p}\left(R^{N}\right), \quad 1 \leq i \leq N\right\}, \\
\text { where } q \geq p \quad \text { if } \quad p \geq N \geq 2, \quad \text { and } \\
p \leq q \leq p^{*}=\frac{N p}{N-p}, \quad \text { when } N>q .
\end{gathered}
$$

When $p=q=2,(1.1)$ is derived by considering the standing waves of the nonlinear Schrödinger equation

$$
i \Phi_{t}=\Delta \Phi+g(|\Phi|) \Phi
$$

where $\Phi \in \mathbf{C}, \Delta=\sum_{i=1}^{N}\left(\partial^{2} / \partial x_{i}^{2}\right), x=\left(x^{1}, x^{2}, \ldots, x^{N}\right) \in R^{N}$. A standing wave of (1.2) is a solution of (1.2) which has the form $\Phi(x, t)=e^{i \beta t} u(x)$; thus $u$ satisfies

$$
\Delta u+g(|u|) u+\beta u=0,
$$

which is a special case of (1.1). For more details about scalar field equations see e.g. $[\mathrm{BL}]$.

Throughout this paper, we denote by $\|u\|_{s}$ the $L^{s}$-norm of the function $u$ over $R^{N}$ and $\|u\|_{s(|x| \geq R)}$ the $L^{s}$-norm of $u$ over the set $\left\{x \in R^{N}|| x \mid \geq R\right\}$ where $s>1$. Let $p, q$, and the space $E$ be given as in (1.1). The norm in $E$ is defined by $\|u\|_{E}=\|\mid \nabla u\|_{p}+\|u\|_{q}$ for any $u \in E$. It is clear the $\left\langle E,\|\cdot\|_{E}\right\rangle$ is a reflexive Banach space.

By Nirenberg's inequality (see [N]), $E$ is imbedded in $L^{t}\left(R^{N}\right)$ for $t \geq q$ when $N \leq p$ and for $q \leq t \leq p^{*}=N p /(N-p)$ when $N>p$. Furthermore, we have the following result which is a generalization of $N$. Trudinger's inequality (see $[\mathrm{L}]$ ).

Supported by the Youth Foundation of the National Science Foundation of China. 
Lemma 1.3. Suppose that $0<\gamma<N /(N-1), r \geq q \geq N$ with $\gamma n_{0}>$ $r+N /(N-1), b>0$ where $n_{0}$ is a positive integer. Then for all $u \in E$

$$
\sum_{n=n_{0}}^{+\infty} \frac{b^{n}}{n !} \int_{R^{N}}|u|^{\gamma n} d x \leq C\left(\||\nabla u|\|_{N}\right)\|u\|_{r}^{r}
$$

and for $0<\tau<1$

$$
\sum_{n=n_{0}}^{+\infty} \frac{1}{n !}\left(\int_{R^{N}}|u|^{\gamma n} d x\right)^{\tau} \leq \tilde{C}\left(\||\nabla u|\|_{N}\right)\|u\|_{r}^{r \tau}
$$

where $C(t), \tilde{C}(t)$ are nonnegative real functions on $[0,+\infty)$. Furthermore for each $M>0$, there is a constant $K(M)>0$ such that

$$
C\left(\||\nabla u|\|_{N}\right) \leq K(M), \quad \tilde{C}\left(\|\mid \nabla u\|_{N}\right) \leq K(M)
$$

whenever $\||\nabla u|\|_{N} \leq M$ and furthermore there is a constant $K$ such that

$$
C\left(\||\nabla u|\|_{N}\right) \leq K\||\nabla u|\|_{N}^{\gamma n_{0} a_{n_{0}}}
$$

whenever $\||\nabla u|\|_{N} \leq 1$. Here $a_{n_{0}}$ is a positive constant depending only on $n_{0}$.

Proof. This lemma was proved in [LZ, Lemma 1], but for completeness, we sketch the proof.

By the results of C. Talenti (see [T]), we know that if $s, t>1,1 / s=1 / t-1 / N$ and when $|\nabla h| \in L^{t}\left(R^{N}\right)$, then

$$
\|h\|_{s} \leq K(N, t)\||\nabla h|\|_{t}
$$

where

$$
K(N, t)=\frac{t-1}{N-t}\left[\frac{N-t}{N(t-1)}\right]^{1 / t}\left[\frac{\Gamma(N+1)}{\Gamma(N / t) \Gamma(N+1-N / t) \omega_{N-1}}\right]^{1 / N}
$$

and $\omega_{N-1}=\pi^{N / 2} / \Gamma\left(\frac{1}{2} N+1\right)$.

Setting $a_{n}=1-r / \gamma n\left(n \geq n_{0}\right), h=|u|^{1 / a_{n}}$ and using (1.5) and Hölder's inequality we obtain, for each $u \in E$,

$$
\int_{R^{N}}|u|^{\gamma n} d x \leq\left[\frac{K\left(N, \mu_{n}\right)}{a_{n}}\right]^{\gamma n a_{n}}\|\mid \nabla u\|_{N}^{\gamma n a_{n}}\|u\|_{r}^{r}
$$

where $\mu_{n}$ is such that $1 / \gamma n a_{n}=1 / \mu_{n}-1 / N$. On the other hand, it is easy to see that

$$
K\left(N, \mu_{n}\right) \leq C n^{(N-1) / N}
$$


where $C>0$ is a constant independent of $n$, so we have

$$
\sum_{n=n_{0}}^{+\infty} \frac{b^{n}}{n !} \int_{R^{N}}|u|^{\gamma n} d x \leq \sum_{n=n_{0}}^{+\infty} \frac{b^{n}}{n !} C^{\gamma n a_{n}} n^{(N-1) \gamma n a_{n} / N}\|\mid \nabla u\|_{N}^{\gamma n a_{n}}\|u\|_{r}^{r},
$$

from which the lemma follows.

Next, we state the conditions imposed on $c(x)$ and $f(x, t)$ in (1.1).

$\left(c_{1}\right)$ The function $c(x)$ belongs to $C^{0}\left(R^{N}, R^{1}\right)$, and there is a constant $c>0$ such that $c(x) \geq c$ for any $x \in R^{N}$.

$\left(f_{1}\right) \quad f(x, t) \in C^{0}\left(R^{N} \times R^{1}, R^{1}\right)$.

$\left(f_{2}\right) \lim _{t \rightarrow 0} f(x, t) /|t|^{q-1}=0$ uniformly in $x \in R^{N}$.

$\left(f_{3}\right)$ If $N<p$, then there is a $l, q<l<+\infty$ such that $\lim _{t \rightarrow \infty} f(x, t) /|t|^{l-1}=0$ uniformly in $x \in R^{N}$.

If $N=p$, then there is a $\gamma$ with $0<\gamma<N /(N-1)$ such that

$$
\lim _{t \rightarrow \infty} f(x, t) / e^{|t|^{\gamma}}=0 \quad \text { uniformly in } x \in R^{N} .
$$

If $N>p$, then there is a constant $b \geq 0$ such that

$$
\lim _{t \rightarrow \infty} f(x, t) /|t|^{p^{*}-1}=b \quad \text { uniformly in } x \in R^{N} .
$$

Under the above conditions $\left(c_{1}\right),\left(f_{1}\right)-\left(f_{3}\right)$, we easily see that for any $\varepsilon>0$, there is a $C_{\varepsilon}>0$ such that

$$
\begin{gathered}
|f(x, t)| \leq \varepsilon|t|^{q-1}+C_{\varepsilon}|t|^{p^{*}-1}, \text { for all }(x, t) \in R^{N} \times R^{1} \text { if } N>p \\
|f(x, t)| \leq \varepsilon|t|^{q-1}+C_{\varepsilon}|t|^{l-1}, \text { for all }(x, t) \in R^{N} \times R^{1} \text { if } N<p, \\
|f(x, t)| \leq \varepsilon|t|^{q-1}+C_{\varepsilon} \sum_{n=n_{0}}^{+\infty} \frac{|t|^{\gamma n+N-1}}{n !}, \text { for all }(x, t) \in R^{N} \times R^{1} \text { if } N=p .
\end{gathered}
$$

A function $u \in E$ is called a weak solution of (1.1) if for each $v \in E$

$$
\int_{R^{N}}\left[\sum_{i=1}^{N}|\nabla u|^{p-2} \frac{\partial u}{\partial x_{i}} \frac{\partial v}{\partial x_{i}}+c(x)|u|^{q-2} u v-f(x, u) v\right] d x=0 .
$$

Note that under conditions $\left(c_{1}\right),\left(f_{1}\right)-\left(f_{3}\right), u \equiv 0$ is always the trivial solution of (1.1). The existence of nontrivial weak solutions of (1.1) was studied in [BL] for $p=q=2$ and in [Li], [LZ], and [YZ] for general $q \geq p$.

It is our aim in this paper to study some properties of the weak solutions of (1.1). The main result is the following: 
Theorem 1.11. Suppose that $\left(c_{1}\right),\left(f_{1}\right)-\left(f_{3}\right)$ hold and $u \in E$ is a weak solution of (1.1). Then $u \in L^{\infty}\left(R^{N}\right)$ and there is a $t>1, R_{0}=R_{0}(t)>0$ such that for any $R \geq R_{0}$

$$
\|u\|_{\infty(|x| \geq R)} \leq C\|u\|_{t(|x| \geq R / 2)}<+\infty
$$

where $C$ is a positive constant independent of $R$. Furthermore, $\lim _{|x| \rightarrow \infty} u(x)$ $=0$, and $u \in C_{\mathrm{loc}}^{1, \alpha}\left(R^{N}\right)$ for some $0<\alpha<1$.

Remark 1.13. $C^{1, \alpha}$ regularity is the best possible in general for weak solutions of degenerate elliptic equations like (1.1) as one can see from a simple example in $[\mathrm{To}]$.

There have been some results for $C^{1, \alpha}$ regularity of weak solutions in bounded domains (see e.g. [LU], [To]). For the unbounded domain $R^{N}, \mathrm{H}$. Brezis and E.H. Lieb showed that weak solutions of semilinear elliptic systems, in particular of (1.1) when $N>p=2$, are in $L^{\infty}\left(R^{N}\right) \cap C_{\text {loc }}^{1, \alpha}\left(R^{N}\right)$ for all $0<\alpha<1$ and the solutions tend to zero as $|x| \rightarrow+\infty$ (see [BLi]). But their method seems not to extend to $p \neq 2$.

The main difficulty in proving Theorem 1.11 is to prove (1.12) and $u \in$ $L^{\infty}\left(R^{N}\right)$. We overcome this difficulty by using the Nash-Moser method (see $[\mathrm{GT}])$ together with careful estimates.

\section{Proof of the main theorem}

In this section, we prove the main result of this paper, Theorem 1.11. By the main result in [To], we need only to prove (1.12) and $u \in L^{\infty}\left(R^{N}\right)$ together with $\lim _{|x| \rightarrow \infty} u(x)=0$.

Suppose that $u$ is a weak solution of (1.1). For any $R>0,0<r \leq R / 2$, let $\eta \in C^{\infty}\left(R^{N}\right), 0 \leq \eta \leq 1$ with

$$
\eta=\left\{\begin{array}{ll}
1 & \text { if }|x| \geq R \\
0 & \text { if }|x| \leq R-r,
\end{array} \quad|\nabla \eta| \leq 2 / r\right.
$$

We set $u^{+}=\max (0, u), u_{L}^{+}=\min \left(u^{+}, L\right)$ where $L>0$.

We first deal with the case where $N>p$. To this end, let $v=\eta^{p} u^{+} u_{L}^{+p(\beta-1)}$, $W_{L}=\eta u^{+} u_{L}^{+\beta-1}$ for any $\beta \geq 1$. Then by (1.7), by the definition of weak solutions and by Sobolev's imbedding, we have, for some constant $C>0$, that, for each $\beta \geq 1$,

$$
\begin{aligned}
\left\|W_{L}\right\|_{p \gamma}^{p} & \leq C \int_{R^{N}}\left|\nabla W_{L}\right|^{p} d x \\
& \leq C \beta^{p}\left(\int_{R^{N}} u^{+p^{*}} \eta^{p} u_{L}^{+p(\beta-1)} d x+\int_{R^{N}}|\nabla \eta|^{p} u^{+p} u_{L}^{+p(\beta-1)} d x\right) .
\end{aligned}
$$


Some properties of weak solutions of nonlinear scalar field equations

We claim that

$$
u \in L^{p^{* 2} / p}(|x| \geq R)
$$

for $R$ large enough. In fact, let $\beta=p^{*} / p$, from (2.2) we have

$$
\begin{aligned}
& \left(\int_{R^{N}}\left(\eta u^{+} u_{L}^{+\left(p^{*}-p\right) / p}\right)^{p^{*}} d x\right)^{p / p^{*}} \\
& \leq C(N, p)\left\{\left[\int_{R^{N}}\left(\eta u^{+} u_{L}^{+\left(p^{*}-p\right) / p}\right)^{p^{*}} d x\right]^{p / p^{*}}\left(\int_{|x| \geq R-r} u^{+p^{*}} d x\right)^{\left(p^{*}-p\right) / p}\right. \\
& \left.\quad+\int_{R^{N}}|\nabla \eta|^{p} u^{+p} u_{L}^{+p^{*}-p} d x\right\} \\
& \leq C(N, p)\left\{\left[\int_{R^{N}}\left(\eta u^{+} u_{L}^{+\left(p^{*}-p\right) / p}\right)^{p^{*}} d x\right]^{p / p^{*}}\left\|u^{+}\right\|_{p^{*}\left(|x| \geq \frac{1}{2} R\right)}^{p^{*}-p}\right. \\
& \left.\quad+\int_{R^{N}}|\nabla \eta|^{p} u^{+p} u_{L}^{+\left(p^{*}-p\right)} d x\right\} .
\end{aligned}
$$

Since $u^{+} \in L^{p^{*}}\left(R^{N}\right),\left\|u^{+}\right\|_{p^{*}\left(|x| \geq \frac{1}{2} R\right)}^{p^{*}-p} \leq 1 / C(N, p)$ for $R$ large enough. Hence we obtain

$$
\begin{aligned}
& \left(\int_{|x| \geq R}\left(u^{+} u_{L}^{+\left(p^{*}-p\right) / p}\right)^{p^{*}} d x\right)^{p / p^{*}} \leq\left(\int_{R^{N}}\left(\eta u^{+} u_{L}^{+\left(p^{*}-p\right) / p}\right)^{p^{*}} d x\right)^{p / p^{*}} \\
& \quad \leq C(N, p) \int_{R^{N}}|\nabla \eta|^{p} u^{+p} u_{L}^{+\left(p^{*}-p\right)} d x \leq \frac{C}{r^{p}} \int_{R^{N}} u^{+p^{*}} d x
\end{aligned}
$$

Thus (2.2) follows.

Next, we note that if $\beta=p^{*}(t-1) / p t$ with $t=p^{* 2} /\left(p^{*}-p\right) p$, then $\beta>1$ and $p t /(t-1)<p^{*}$. Now suppose that $u^{+} \in L^{p \beta t /(t-1)}(|x| \geq R-r)$ for some $\beta \geq 1$. Then (2.3) gives that

$$
\begin{aligned}
\left\|W_{L}\right\|_{p^{*}}^{p} \leq & C \beta^{p}\left\{\left[\int_{|x| \geq R-r}\left(\eta^{p} u^{+p \beta}\right)^{t /(t-1)} d x\right]^{1-1 / t}\right. \\
& \times\left(\int_{|x| \geq R-r} u^{+\left(p^{*}-p\right) t} d x\right)^{1 / t} \\
& \left.+\frac{\left[R^{N}-(R-r)^{N}\right]^{1 / t}}{\gamma p}\left(\int_{|x| \geq R-r} u^{+p \beta t /(t-1)} d x\right)^{1-1 / t}\right\} \\
\leq & C \beta^{p}\left(1+\frac{R^{N / t}}{r^{p}}\right)\left(\int_{|x| \geq R-r} u^{+p \beta t /(t-1)} d x\right)^{1-1 / t} .
\end{aligned}
$$


Letting $L \rightarrow+\infty$ in (2.5), we obtain

$$
\left\|u^{+}\right\|_{\beta p^{*}(|x| \geq R)}^{p \beta} \leq C \beta^{p}\left(1+\frac{R^{N / t}}{r^{p}}\right)\left\|u^{+}\right\|_{p \beta t /(t-1)(|x| \geq R-r)}^{p \beta} .
$$

If we set $\chi=p^{*}(t-1) / p t, s=p t /(t-1)$, then

$$
\left\|u^{+}\right\|_{\beta \chi s(|x| \geq R)} \leq C^{1 / \beta} \beta^{1 / \beta}\left(1+\frac{R^{N / t}}{r^{p}}\right)^{1 / p \beta}\left\|u^{+}\right\|_{\beta s(|x| \geq R-r)} .
$$

Let $\beta=\chi^{m},(m=1,2, \ldots)$, then we get

$$
\left\|u^{+}\right\|_{\chi^{m+1} s(|x| \geq R)} \leq C^{\chi^{-m}} \chi^{m \chi^{-m}}\left(1+\frac{R^{N / t}}{r^{p}}\right)^{1 / p \chi^{m}}\left\|u^{+}\right\|_{\chi^{m} s(|x| \geq R-r)} .
$$

It is clear that $p>N / t$. So if $r_{m}=2^{-(m+1)} R$, then (2.6) implies

$$
\begin{aligned}
& \left\|u^{+}\right\|_{\chi^{m+1} s(|x| \geq R)} \leq\left\|u^{+}\right\|_{\chi^{m+1} s\left(|x| \geq R-r_{m+1}\right)} \\
& \leq C \sum_{i=1}^{m} \chi^{-i} \chi^{\sum_{i=1}^{m} i \chi^{-i}} \exp \left(\sum_{i=1}^{m} \ln \left(1+2^{p(i+1)}\right) / p \chi^{i}\right)\left\|u^{+}\right\|_{\chi s\left(|x| \geq R-r_{1}\right)} \\
& \leq C\left\|u^{+}\right\|_{p^{*}\left(|x| \geq \frac{1}{2} R\right)} .
\end{aligned}
$$

Letting $m \rightarrow+\infty$ in the last inequality, we obtain

$$
\left\|u^{+}\right\|_{\infty(|x| \geq R)} \leq C\left\|u^{+}\right\|_{p^{*}\left(|x| \geq \frac{1}{2} R\right)} .
$$

Similarly, we can show

$$
\left\|u^{-}\right\|_{\infty(|x| \geq R)} \leq C\left\|u^{-}\right\|_{p^{*}\left(|x| \geq \frac{1}{2} R\right)}
$$

where $u^{-}=\max (-u, 0)$; hence (1.12) holds for $N>p$ and $\lim _{|x| \rightarrow \infty} u(x)=0$.

To show that $\|u\|_{\infty}<+\infty$ when $N>p$, we need only show that for any $x_{0} \in$ $R^{N}$, there is a ball $B_{R}\left(x_{0}\right)=\left\{x \in R^{N}|| x-x_{0} \mid \leq R\right\}$ such that $\|u\|_{\infty\left(B_{R}\left(x_{0}\right)\right)}<$ $+\infty$. But this was essentially done in $[\mathrm{ZY}]$ for weak solutions of equations similar to (1.1) in bounded domains. We just sketch the proof of this fact.

For any $x_{0} \in R^{N}, R>0,0<r \leq \frac{1}{2} R$, let $\xi \in C_{0}\left(R^{N}\right)$ with $0 \leq \xi \leq 1$ and

$$
\xi= \begin{cases}1 & \text { if }\left|x-x_{0}\right| \leq R \\ 0 & \text { if }\left|x-x_{0}\right| \geq R+r\end{cases}
$$

and $|\nabla \xi| \leq 2 / r$. Write $\bar{v}=\xi^{p} u^{+} u_{L}^{+p(\beta-1)}, \bar{W}_{L}=\xi u^{+}\left(u_{L}^{+}\right)^{-1}$, we can show that for $R_{0}$ small enough

$$
u^{+} \in L^{p^{* 2} / p}\left(B_{R}\left(x_{0}\right)\right)
$$


and similarly for some $\bar{R}$ that $u^{+} \in L^{\infty}\left(B_{\bar{R}}\left(x_{0}\right)\right)$ by the method used above. Thus $\left\|u^{+}\right\|_{\infty}<+\infty$ hence $\|u\|_{\infty}<+\infty$ and we have completed the proof of Theorem 1.11 in the case $N>p$.

If now $N=p$, we set $v=u^{+} u_{L}^{+N(\beta-1)},(\beta \geq 1)$; then $v \in E$ and the definition of weak solutions gives that

$$
\begin{aligned}
\int_{R^{N}}\left|\nabla u^{+}\right|{ }^{N} u_{L}^{+N(\beta-1)} d x+N(\beta-1) \int_{R^{N}}\left|\nabla u_{L}^{+}\right|^{N} u_{L}^{+N(\beta-1)} d x \\
\quad+c \int_{R^{N}} u^{+q} u_{L}^{+N(\beta-1)} d x \\
\leq C \sum_{n=n_{0}}^{+\infty} \frac{1}{n !} \int_{R^{N}} u^{+\gamma n+N-1} u^{+} u_{L}^{+N(\beta-1)} d x
\end{aligned}
$$

If we set $W_{L}=u^{+} u_{L}^{+\beta-1}$, then (2.7) implies

$$
\int_{R^{N}}\left|\nabla W_{L}\right|^{N} d x \leq C \beta^{N} \sum_{n=n_{0}}^{+\infty} \frac{1}{n !} \int_{R^{N}} u^{+\gamma n}\left|W_{L}\right|^{N} d x
$$

Using Hölder's inequality, we get

$$
\left\|\left|\nabla W_{L}\right|\right\|_{N}^{N} \leq C \beta^{N} \sum_{n=n_{0}}^{+\infty} \frac{1}{n !}\left(\int_{R^{N}} u^{+\left(\gamma+\varepsilon_{0}\right) n} d x\right)^{\gamma /\left(\gamma+\varepsilon_{0}\right)}\left\|W_{L}^{N}\right\|_{t / N}
$$

where $\varepsilon_{0}$ is small enough such that $\gamma+\varepsilon_{0}<N /(N-1), t=N\left(\gamma+\varepsilon_{0}\right) / q_{0} \geq q$. Thus Lemma 1.3 yields

$$
\left\|\left|\nabla W_{L}\right|\right\|_{N} \leq C \beta\left\|W_{L}\right\|_{t} .
$$

Hence by Nirenberg's inequality (see [N]) there is a $s>t$ with

$$
\left\|W_{L}\right\|_{s} \leq C\left(\left\|\left|\nabla W_{L}\right|\right\|_{N}+\left\|W_{L}\right\|_{t}\right) \leq C \beta\left\|W_{L}\right\|_{t}
$$

where $C>0$ is a constant from which we obtain $\left\|u^{+}\right\|_{\infty}<+\infty$ by standard Nash-Moser iteration. Similarly $\left\|u^{-}\right\|_{\infty}<+\infty$ and hence $\|u\|_{\infty}<+\infty$.

To show (1.12) for $N=p$, we can use the same method we used in the case where $N>p$. In fact, let $v=\eta^{N} u^{+} u^{+N(\beta-1)}, W_{L}=\eta u^{+} u_{L}^{+\beta-1}$ where $\eta$ was given by (2.1) for $\frac{1}{2} R \geq r>0$, then by the definition of weak solutions and Lemma 1.3 we have, for any $\varepsilon>0$, that

$$
\int_{R^{N}}\left|\nabla u^{+}\right|^{N} \eta^{N} u_{L}^{+N(\beta-1)} d x
$$




$$
\begin{aligned}
& +N \int_{R^{N}} \sum_{i=1}^{N}\left|\nabla u^{+}\right|^{N-2} \frac{\partial u^{+}}{\partial x_{i}} \frac{\partial \eta}{\partial x_{i}} \eta^{N-1} u^{+N(\beta-1)} u^{+} d x \\
& +N(\beta-1) \int_{R^{N}}\left|\nabla u_{L}^{+}\right|^{N} \eta^{N} u_{L}^{+N(\beta-1)} d x+c \int_{R^{N}} u^{+q} \eta^{N} u^{+N(\beta-1)} d x \\
& \leq \int_{R^{N}} f\left(x, u^{+}\right) u^{+} \eta^{N} u_{L}^{+N(\beta-1)} d x \\
& \leq \varepsilon \int_{R^{N}} u^{+q} \eta^{N} u_{L}^{+N(\beta-1)} d x+C_{\varepsilon}\left\|W_{L}\right\|_{t}^{N}
\end{aligned}
$$

for some $C_{\varepsilon}>0$ and $t>q$.

Taking $\varepsilon>0$ small enough and using Young's inequality and $\left(c_{1}\right)$, we get

$$
\begin{aligned}
& \int_{R^{N}}\left|\nabla u^{+}\right|^{N} \eta^{N} u_{L}^{+N(\beta-1)} d x+N(\beta-1) \int_{R^{N}}\left|\nabla u_{L}^{+}\right|^{N} \eta^{N} u_{L}^{+N(\beta-1)} d x \\
& \quad+\bar{c} \int_{R^{N}} u^{+q} \eta^{N} u_{L}^{+N(\beta-1)} d x \\
& \leq c\left\|W_{L}\right\|_{t}^{N}+N \int_{R^{N}}\left|\nabla u^{+}\right|^{N-1}|\nabla \eta| \eta^{N-1} u^{+} u_{L}^{+N(\beta-1)} d x \\
& \leq \delta \int_{R^{N}}\left|\nabla u^{+}\right|^{N} \eta^{N} u_{L}^{+N(\beta-1)} d x \\
&+C_{\delta} \int_{R^{N}}|\nabla \eta|^{N} u^{+N^{*}} u_{L}^{+N(\beta-1)} d x+C\left\|W_{L}\right\|_{t}^{N}
\end{aligned}
$$

where $\delta>0$ is arbitrary and $\bar{c}>0$ is a constant.

Choosing $\delta>0$ small enough, we have

$$
\begin{aligned}
\int_{R^{N}}\left|\nabla u^{+}\right|{ }^{N} \eta^{N} u_{L}^{+N(\beta-1)} d x+N(\beta-1) \int_{R^{N}}\left|\nabla u_{L}^{+}\right|^{N} \eta^{N} u_{L}^{+N(\beta-1)} d x \\
\quad+\tilde{C} \int_{R^{N}} u^{+q} \eta^{N} u_{L}^{+N(\beta-1)} d x \\
\leq C\left(\left\|W_{L}\right\|_{t}^{N}+\int_{R^{N}}|\nabla \eta|^{N} u^{+} u_{L}^{+N(\beta-1)} d x\right)
\end{aligned}
$$

where $C>0, \tilde{C}>0$ are constants. Hence

$$
\begin{aligned}
& \int_{R^{N}}\left|\nabla W_{L}\right|^{N} d x+\left\|W_{L}\right\|_{t}^{N} \\
& \quad \leq c\left[\int_{R^{N}}|\nabla \eta|^{N} u^{+N} u_{L}^{+N(\beta-1)} d x+\int_{R^{N}} \eta^{N} u_{L}^{+N(\beta-1)}\left|\nabla u^{+}\right|^{N} d x\right.
\end{aligned}
$$


Some properties of weak solutions of nonlinear scalar field equations

$$
\begin{aligned}
& \left.+(\beta-1)^{N} \int_{R^{N}} \eta^{N} u_{L}^{+N(\beta-1)}\left|\nabla u_{L}^{+}\right|^{N} d x\right]+\left\|W_{L}\right\|_{t}^{N} \\
\leq & C \beta^{N}\left(\int_{R^{N}}|\nabla \eta|^{N} u^{+N} u_{L}^{+N(\beta-1)} d x+\left\|W_{L}\right\|_{t}^{N}\right) \\
\leq & C \beta^{N}\left\{1+\frac{\left[R^{N}-(R-r)^{N}\right]^{(t-N) / t}}{\gamma N}\right\}\left\|u^{+} u_{L}^{+\beta-1}\right\|_{t(|x| \geq R-r)}^{N} .
\end{aligned}
$$

Again, by Nirenberg's inequality, for some $s>t$, we have

$$
\begin{aligned}
\left\|W_{L}\right\|_{s} & \leq C\left(\left\|\left|\nabla W_{L}\right|\right\|_{N}+\left\|W_{L}\right\|_{t}\right) \\
& \leq C \beta\left[1+\frac{R^{N(t-N) / t}}{r^{N}}\right]^{1 / N}\left\|u^{+} u_{L}^{+\beta-1}\right\|_{t(|x| \geq R-r)}
\end{aligned}
$$

Letting $L \rightarrow+\infty$, we get

$$
\left\|u^{+}\right\|_{s \beta(|x| \geq R)} \leq C^{1 / \beta} \beta^{1 / \beta}\left[1+\frac{R^{N(t-N) / t}}{\gamma N}\right]^{1 / N \beta}\left\|u^{+}\right\|_{t \beta(|x| \geq R-r)}
$$

where $C$ is a positive constant independent of $R$ and $r$. Let $\chi=s / t, \beta=\chi^{m}$ and $r_{m}=2^{-(m+1)} R$ for $R>1$. Now we obtain

$$
\left\|u^{+}\right\|_{\chi^{m+1} t\left(|x| \geq R-r_{m+1}\right)} \leq C^{\chi^{-m}} \chi^{m \chi^{-m}}\left(1+2^{N(m+2)}\right)^{\chi^{-m} / N}\left\|u^{+}\right\|_{\chi^{m} t(|x| \geq R)}
$$

from which (1.12) follows easily and hence $\lim _{|x| \rightarrow \infty} u(x)=0$.

The case where $N<p$ can be dealt with in the same way; we omit the details. We have thus completed the proof of Theorem 1.11 .

Acknowledgement. The author wishes to express his gratitude to the Department of Mathematics, University of Jyväskylä, and to the Rolf Nevanlinna Institute for financial support during his stay in Jyväskylä in 1989. 


\section{References}

[BL] Berstycki, H., and P.-L. Lions: Nonlinear scalar field equations, I and II. - Arch. Rational Mech. Anal. 82, 1983, 313-336, 347-376.

[BLi] Brezis, H., and E.H. LIEB: Minimum action solution of some vector field equations. Comm. Math. Phys. 96, 1984, 97-113.

[GT] Gilbarg, D., and N.S. Trudinger: Elliptic partial differential equations of second order. - Springer-Verlag, Heidelberg-New York, 1983.

[LU] Ladyzhenskaya, O.A., and N.N. Uealtseva: Linear and quasilinear elliptic equations. - Academic Press, New York-London, 1968.

[Li] LI GongBAo: The existence of infinitely many solutions of quasilinear elliptic PDE in unbounded domains. - Acta Math. Sci. 9, 1989, 175-188.

[LZ] LI GongbaO and ZHU XIPING: Nontrivial solutions of nonlinear scalar field equations with strong nonlinearity. - Acta Math. Sci. 8, 1988, 431-448.

[L] Lions, P.-L.: The concentration-compactness principle in the calculus of variations; the limit case, part 1. - Rev. Mat. Iberoamer. 1, 1985, 45-120.

[N] Nirenberg, L.: On elliptic partial differential equations. - Ann. Scuola Norm. Sup. Pisa Cl. Sci. (3) 13, 1959, 115-162.

[T] TAlenti, C.: Best constant in Sobolev inequality. - Ann. of Math. 110, 1976, 353-372.

[To] Tolksdorf, P.: Regularity for a more general class of quasilinear elliptic equations. - J. Differential Equations 51, 1984, 126-150.

[YZ] YANG JIANFU and ZHU XIPING: On the existence of nontrivial solutions of a quasilinear elliptic boundary value problem for unbounded domains (I). - Acta Math. Sci. 7, 1979, 341-359.

[ZY] ZHU XIPING and YANG JiANFU: Regularity for quasilinear elliptic equations involving critical Sobolev exponents. - To appear.

Academia Sinica

Wuhan Institute of Mathematical Sciences

P.O. Box 30

Wuhan 430071

People's Republic of China

Received 25 November 1988 